\title{
Holography of the Surface Layer in the Visible Range of Electromagnetic Radiation for Its Geometric Modeling
}

\author{
Evgeny Alexandrovich Belkin *, Vyacheslav Nikolaevich Poyarkov, Oleg Ivanovich Markov \\ Department of Experimental and Theoretical Physics, Faculty of Physics and Mathematics, Orel State University I. S. Turgenev, Orel, Russia \\ Email address: \\ BelkinE.A@yandex.ru(E.A. Belkin), oaobzpp@list.ru(V. N. Poyarkov), O.I.Markov@mail(V. N. Poyarkov) \\ ${ }^{*}$ Corresponding author

\section{To cite this article:} \\ Evgeny Alexandrovich Belkin, Vyacheslav Nikolaevich Poyarkov, Oleg Ivanovich Markov. Holography of the Surface Layer in the Visible \\ Range of Electromagnetic Radiation for Its Geometric Modeling. International Journal of Science, Technology and Society. \\ Vol. 6, No. 5, 2018, pp. 72-77. doi: 10.11648/j.ijsts.20180605.11
}

Received: August 22, 2018; Accepted: October 5, 2018; Published: November 6, 2018

\begin{abstract}
One of the main problems of modern measurement technology and Metrology is that no non-destructive testing device, due to its design features, allows to make metrological measurements necessary for the construction of a threedimensional geometric model of the part surface, which is a superposition of the geometric image of the surface and the topography of its microrelief. As a rule, in the calculation of the forming surface of the tool there is no calculation of the topography of its microrelief. This is due to the lack of sufficient information about the geometric structure of the microrelief as a three-dimensional image, due to the use of one-dimensional evaluation parameter. Application for geometric modeling of the microrelief shape of a one-dimensional evaluation parameter-the height of the microrelief, gives an idea of the microrelief as a surface with numerical marks. In the description of the surface with numerical marks, the curvature in the local neighborhood of the given point is not determined, which makes it impossible to construct its full geometric image. The solution to the problem is to create a non-destructive testing device-an optical profilograph, the design of which would allow to measure the geometric characteristics of the surface of the part necessary for structuring its full geometric image and the development of a new geometric approach that allows to obtain this complete geometric image of the part. Installation - optical profilograph refers to measuring equipment, in particular to devices for roughness control. This installation is designed as a complex of non-destructive testing devices of new generation, which is aimed at solving the actual problem in the conduct of metrological measurements required to build a three-dimensional geometric model of the surface of the part, which would be a superposition of the geometric image of the surface of the part and the topography of its microrelief. The principle of operation of the installation is that the holographic image of the part, the scanning indicator of the electromagnetic field are removed cards, which are fixed microrelief profiles of the surface layer, profiles of internal and external surfaces of the part. With these profiles remove the geometric characteristics, which are based on the modular geometric approach allows you to structure the topography of the surface layer microrelief, as well as the internal and external geometry of the surfaces of the part, having a complex shape.
\end{abstract}

Keywords: Optical Profilograph Plant, Optical Circuits of Leith-Upatnieks and Denisyuk, Surface Layer Topography

\section{Assessment of Need in Development of New Instrument Base for Nondestructive Check}

At present technologies and equipment used for the check of both a structure, and geometry of different material parts machined in the course of a manufacturing cycle are based on regulations of those scientific subjects the study objects of which are directly separate elements of the processes mentioned. There is no approach which would allow taking into account processing factors both in the analytical description of a microrelief formation process, and at the immediate nondestructive check of outer and inner geometry of parts in the course of manufacturing reasoning from a single axiomatics.

The necessity to take into account $3 \mathrm{D}$ characteristics of a surface layer micro-relief is particularly urgent at 
manufacturing parts with specified operation properties. It is well-known, that wear-resistance, fatigue strength and other operation properties are defined to a great extent by the form of a surface microrelief.

A part machining process is a single closed structure. One of the results of this process is a formed topography of a surface layer microrelief in a part operation surface.

At the moment the microrelief topography of a part is assessed, as a rule, after its machining, but not in the course of a microrelief formation. Engineering geometry methods allowing the connection of a surface curvature with its contact surface are developed, but not used in view of the absence of a tool basis [1]. Therefore at the geometrical presentation of a surface layer microrelief there is no possibility to restore its structure with the sufficient accuracy essential for the prediction of its topography and for its geometric parameter control during parts machining.

In such a way the development of technology and devices for $3 \mathrm{D}$ nondestructive check of the formation of a surface layer, a structure and parts geometry and also for the assessment of a surface layer microrelief topography is urgent which will allow assessing finally not only quality and control fulfillment, but producing parts with properties and a structure type pre-specified.

As consumers may be enterprises of "Rostech" corporation, and, first of all, "Precise Systems" contours and "Techmach".

An optical profilograph plant for 3D control of complex part dimensions for mechanical engineering must carry out manufacturing procedures having scientific -engineering novelty allowing the definition of a market application and the assurance of the development and manufacture products with new quality and a high level of effectiveness.

Devices for the nondestructive check of surface geometrical characteristics in a part and its microrelief used in industrial production do not allow defining the parameter numerical values for the formation of microrelief topography. That is why among the parts having the same roughness of surface microrelief topography may differ greatly. During the operation it results in life decrease, rapid wear, operation characteristics changes, performance decrease in units and assemblies where this part functions as a constituent.

The used devices for nondestructive check have a number of considerable drawbacks:

Probes investigate an object-part itself and its surface and, at the same time, they interact with it. It results in a low accuracy in metrological measurements.

Optical devices carrying out methods of interferometry, holographic immersion and so on gave information on the basis of which it is possible to form a 3D insufficiently complete geometrical model of the part. In particular, it concerns parts having aerohydrodynamic surfaces.

Scanning devices allow obtaining an idea of a microrelief in a local field without taking into account a curvature of its troughs and peaks.

Devices checking defects in a part form are, as a rule, single-purpose. Some of them investigate outer geometry, others - inner one, the thirds define inner defects. Besides, devices vary in a possibility to control parts with a round shape, those having planar faces, and those with framed discrete-definite surfaces. There is no universal devicewhich would allow carrying out metrological measurements without regard to dimensions and a shape of a part.

The design drawbacks of devices for a nondestructive check limit their functional potentialities. As a rule, a design of devices for control allows measuring numerically onedimensional estimating parameter-height of a microroughness at the investigations of surface roughness and stating a surface profile in a flat section.

A consequence of this is that:

In the computation of a tool shaping surface there is no computation of its microrelief topography. It is connected with the absence of sufficient information on a microrelief geometrical structure as a 3D image in connection with the use of one-dimensional estimating parameter. The application of one-dimensional estimating parameter-height of micro-irregularity for the geometrical modeling of a microrelief form gives an idea of a microrelief as a surface with numerical marks. In the description of a surface with numerical marks a curvature is not defined in a local neighborhood of a given point.

Besides, the uncertainty of surface geometry between sections does not give a possibility to create its complete geometrical image.

In such a way, a problem is that no one modern device for the nondestructive check because of its design peculiarities allows fulfilling metrological measurements essential for the formation of the 3D geometrical model of a part surface which is a super-position of a geometrical image of a surface and a topography of its microrelief.

This problem is urgent at manufacturing parts to operation properties of which there are made high demands in contact surface tribo-conjugations. It also belongs to blade machines operated in hostile gas and liquid environment. It is wellknown that wear-resistance, fatigue strength and other operation properties in tribo-conjugations and also cavitation wear are defined through a surface geometry and topography of a surface microrelief.

A problem solution is in the development of nondestructive check devices of a new generation which allow carrying out metrological measurements essential for the application of a module-geometrical approach to a structure of 3D geometric models of a surface.

\section{Optical Profilograph Plant}

An optical profilograph plant [1] is a complex of systems including laser optical electromechanical and microprocessor subsystems. An aggregate work of these systems under controller management allows carrying out 3D precise holographic control of complex-profile parts.

With an error of not more than $315 \mathrm{~nm}$ (red light) at the operation in the visible range [2], at that, the control before and after processing and also during processing is carried out in a discrete way at different stages. 
A plant table (Figure 1) is installed upon gas absorbers. It has systems for the suppression of low-frequency, mid-range and high-frequency mechanical oscillations. The table is supported by lifting jacks of an original design which allow changing its height. Optical systems for recording and holographic image playback are installed arbitrarily, that is, not along a linear guide as it is customary. Mandrels for optic elements: mirrors, micro-lenses, light flow dividers, pin-halls (spatial filters) and so on, have magnetic pillows as a table surface is made of material having high ferro-magnetic properties. Mandrels for optic elements are installed on ball bearings which allow directing them at a certain angle in a plane chosen arbitrarily, that is to say, mandrels have six degrees of freedom. It is difficult to obtain a similar number of freedom degrees at manufacturing common platforms for optical element positioning.

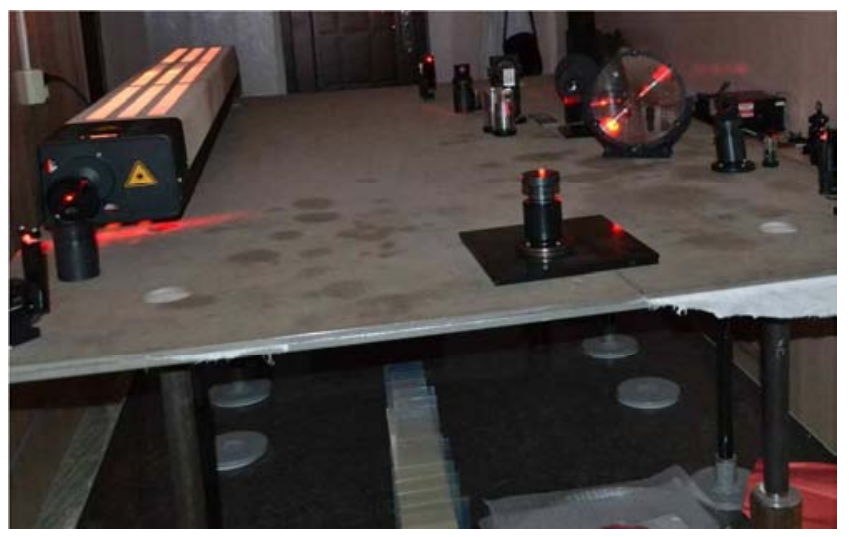

Figure 1. Optic profilograph plant.

An optical profilograph plant [3] must ensure a previous screen presentation (image hanging in air) at the reconstruction for the further obtaining microrelief outline maps of the surface under investigation.

A subsystem obtaining maps from the holographic image of a surface layer represents a mechanical hand. In the mechanical hand there is installed an electromagnetic system which allows fixing a mechanical hand position. On the hand there is strengthened a piezo-nano-positioner. A camcorder with an interface is installed on the positioner.

Maps are transmitted to a computer for surface layer geometrical modeling.

\section{Methods for Surface Layer Holography}

Basic methods for surface layer holography are the following:

Leigth-Upatnieks' method.

Denisyuk's method.

It should be emphasized that to obtain a surface layer holographic image of a part surface having flat faces at different processing methods, that is, roughing, finishing, super-finishing one should choose optical systems for each definite case. There is no universal or flexible optical system suitable for a surface layer holography at different techniques of part processing at the current stage of engineering development. This situation is true also for the holography of the surface layer of parts having surfaces with a complex shape both inner surfaces, and external ones.

For the surface layer holography of flat surfaces and inner ones in parts one can use open optical systems (Figure 2), and for the holography of a surface layer of an external surface with a complex shape it is expedient to use closed optical systems: recording and playback are in a cone or in a cylinder.

When using a microscope for the magnification of the surface under investigation a visible area of a surface layer becomes narrower and the more significant, the more the multiplicity of a microscope magnification is. At the same time, the more the multiplicity of magnification, the lesser becomes the illumination of a visible area. The illumination of the visible area of a surface layer must allow obtaining a holographic image from which it is possible to obtain maps of a microrelief profile. It is possible to achieve through an adjustment of the whole system of holography. At the same time certain difficulties arise, if we take into account that fact that a maximum multiplicity of a microscope is 2500 .

\section{3D Surface Layer Modeling}

The simulation model of a microrelief formation process contains, as a rule, a statistical description of a shaping surface. In case of abrasion it shows how the selection of abrasive grains contacting with the part under processing takes place. A statistical description is expressed through such output values as surface purity processing and material removal intensity. At that, significant concepts are: a distance between active cutting points, a depth to which these points penetrate in a part at chip removal, effects conditioned with elastic deformation and destruction mechanisms which change surfaces of an abrasive tool and a part at their interaction.

Abrasive surface profiles in similar models of a grinding process are considered in sections by a plane parallel to a cutting direction, or by a plane perpendicular to this direction. It is necessary for that to emphasize those statistical properties of a shaping surface which describe directly a chip removal process. Changes in a profile height are considered as values forming a stochastic process, in particular, Markov's chain. A geometrical model in which there is analyzed a topography of a microreliefof a surface layer worked with an abrasive tool and conditioned with its microgeometry, kinematics of an abrasion process, dynamics of a complex motion of abrasive grains fixed in a bunch does not give a complete description of the formation process. The process of a surface microrelief formation which takes place actually in a space cannot be described with $3 \mathrm{D}$ simulation model on the basis of two-dimensional models of its elements.

In fact, reasoning from that the profile of the surface under processing after each operation is repeated well enough and a 
microprofile of any cross-section of a part it is possible to draw the following conclusion: a conditional height of microroughness in the area of a contact is a maximum depth of grain penetration into a surface under grinding. But, at the expense of grain cut covering by width, a length of the contact of each grain with the surface worked in this crosssection of the part is unequal, and the edges of each scratch are located at different levels.

From this it follows that the depth of each cut, that is, the depth of grain penetration into a surface becomes uncertain. That is why it necessitates to assume as a depth of grain penetration in this cross-section a depth calculated from the central line between tips of micro-asperities of the surface obtained, supposing that the edges of grain cutsmove on an average uniformly up and down with regard to a centerline.

In fact, initially, the geometrical theory of a grinding process operates with mean geometrical values: a conditional height of micro-asperity, a depth of grain penetration into the surface under grinding and others. These values do not allow unambiguously presenting a cutting profile of a shaping surface in a fixed plane section and its change in the course of time. From here it is possible to draw a conclusion that the process of a microrelief formation from the point of view of mathematical modeling remains uncertain.

Life increase in wear machinery belongs to the number of the most significant problems of modern mechanical engineering. Functional failures in machine operation, due to parts wear, limit the length of their common operation.

Under wear one implies material surface layer destruction as a result of outer force impacts at friction followed with material softening including those caused with liquid or gas media impact.

For wear decrease in surface layers of conjugations and that of a functional surface of a blade which is caused by a microrelief formed chaotically it should be expedient to reproduce surface layer microgeometry when machining parts in accordance with the specified geometric characteristics.

A microgeometry simulator of conjugation functional surfaces must contain information allowing the computation of micro-engagements which, on the one hand, eliminate considerably a surface layer mechanical destruction and decrease a probability of abrasive particles penetration into conjugations, on the other hand, increase the performance of a kinematic pair at the expense of the decrease of angular micro-deviations of its pivot pins from a design position.

That is why wear-resistance of materials (characteristics of material ability to resist wear under certain conditions of an outer impact) is in a complex tie with their stress-strain and physical-chemical properties. At that, in the simple cases of wear only this tie has an ambiguous character (for instance, a direct dependence between hardness and wear-resistance).

In general, wear-resistance is defined with the total aggregate of processes the generation and development of which depend upon initial properties of materials and conditions of an outer impact.

In such a way, the process of a microrelief formation in a surface layer of a part must be defined by a type of wear process which a part is subjected to at operation. It gives a possibility to control a wear process of a part.

The output characteristics of the formation of a surface microrelief must be geometrical ones which would give an idea of a geometrical structure of the microrelief obtained in 3D space.

Output geometrical characteristics determined on the basis of classical concepts do not present complete information on the micro-geometry of a surface layer after machining. Besides, in most of modern simulators of the process of a microrelief formation the given parameters are determined regardless of the type of part functional surface wear.

If we set a problem of the correspondence of the microrelief topography obtained as a result of part machining in view of its wear, then there is a problem of a microrelief formation with specified geometrical characteristics.

There is one of the ways for widening the potentialities of control devices and for the application of information obtained with their help for the formation of 3D models, the application of system complexes investigating a holographic image of an object. One of such system complexes is a profilograph working in an optical interval of the frequencies of electromagnetic waves.

The design peculiarities of system complexes of this series allow using a module-geometrical approach for modeling the surface under investigation.

In its turn a module-geometrical approach allows obtaining the topography of a microrelief with the analytical description and saving a natural curvature of a surface.

In the second approximation a contacting paraboloid has in the point of a contact with a surface a curvature equal to the curvature of this surface. The analytical presentation of the contacting paraboloid through basic curvatures of a surface in the point of a contact allows defining a tie between a surface curvature change and parameter changes of this analytical presentation (Figure 3). If we consider a microrelief as a surface of a complex shape then as a system of criteria for the assessment of a microrelief topography one should choose a height of micro-unevenness and basic curvatures of a surface. A height of micro-unevenness characterizes the position of a point of a contact regarding the co-ordinates chosen. Basic curvatures of a surface allow making a computation of the analytical presentation of a contacting paraboliod and restoring a local area of a surface in the point of a contact as a part of the surface of a contacting paraboloid.

The offered system of criteria for the assessment of a microrelief allows defining analytically its topography for any kind of a surface (Figure 4) considered in the theory of shaping. A kind of a surface defines only a choice of coordinates where a numerical calculation is performed of $3 \mathrm{D}$ geometrical model of a microrelief.

The independence of the offered system of criteria for the assessment of a microrelief from the kind of the surface under consideration allows setting up a hypothesis of information completeness which is contained in this system. This hypothesis has an experimental confirmation. 


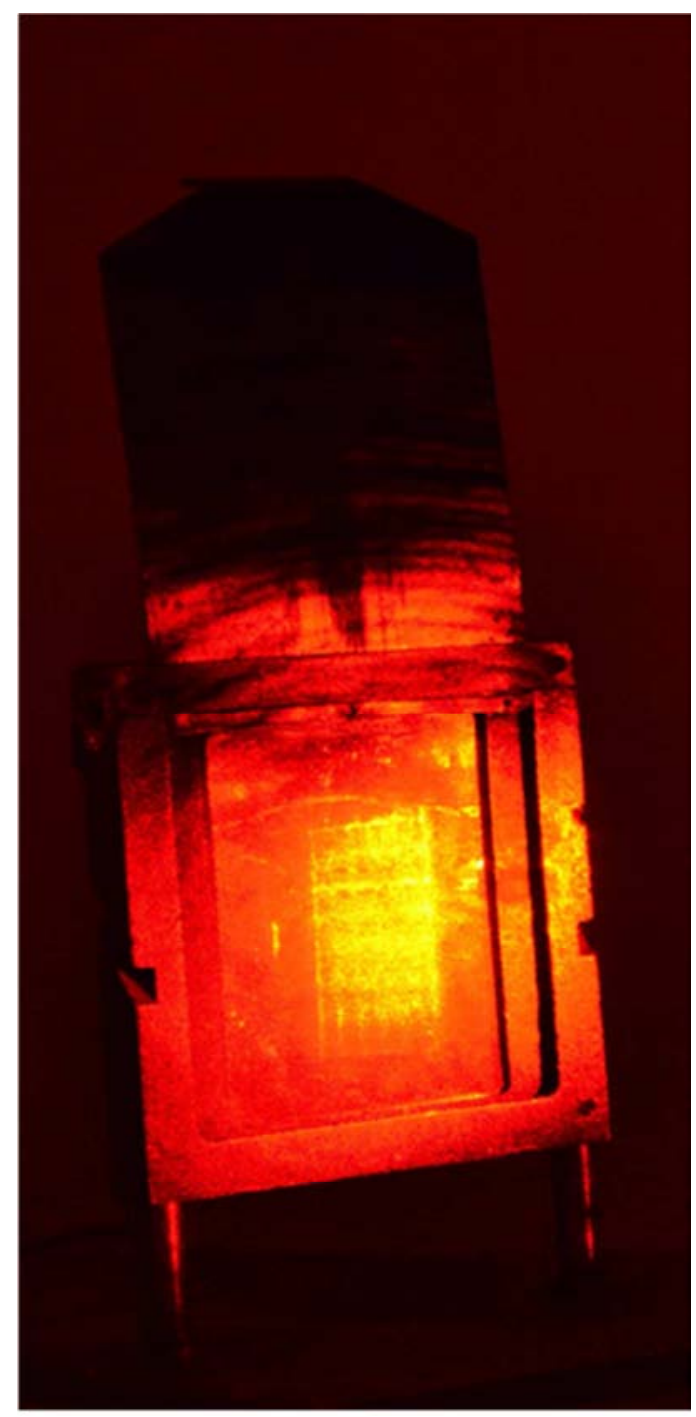

Figure 2. Holographic image of a surface layer after finishing.

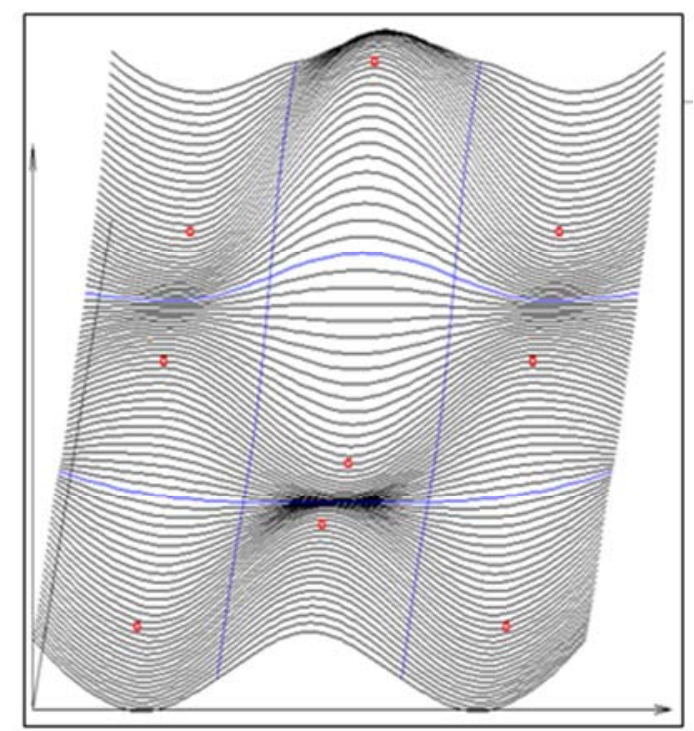

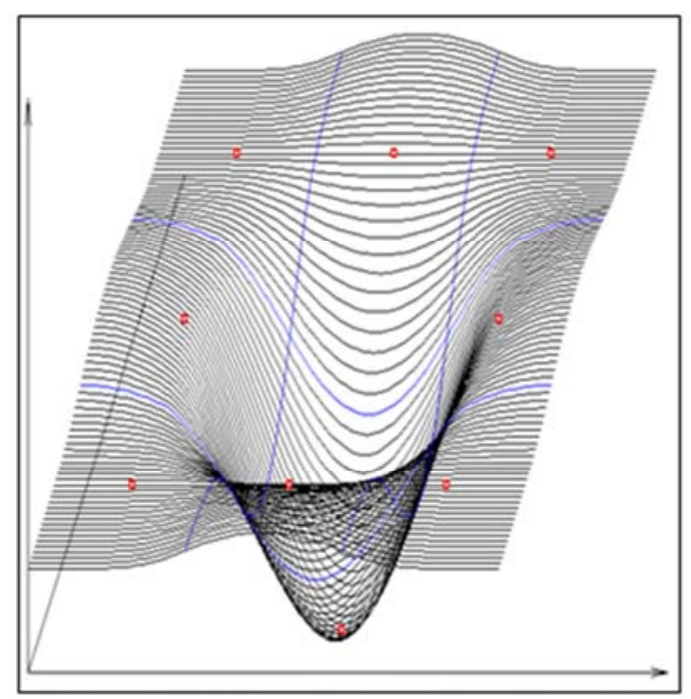

Figure 3. Results of test computations at interpolation of points $3 \times 3$ specified by a grid.

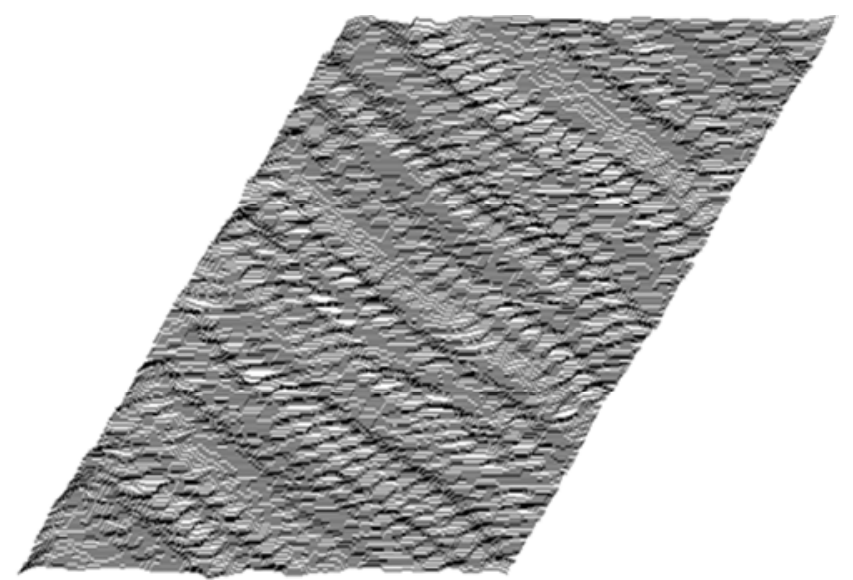

Figure 4. 3D geometric model of a surface layer of a flat facet after finishing.

\section{Application Field of Optical Profilograph Plant}

An optical profilograph plant can be used for a nondestructive check of microrelief topography and geometry of precision parts in machinery and mechanisms.

\section{Conclusions}

1 New generation system complexes for nondestructive check of a microrelief topography formation are developed for application in structuring on the basis of a module-geometrical approach to a microrelief topography, and also to geometry (external, inner) of complex surfaces.

2 There is developed an optical profilograph plant scanning a holographic image of an object in the visible interval of electromagnetic waves which allows obtaining surface characteristics for structuring its $3 \mathrm{D}$ model on the basis of a module-geometrical approach. 


\section{References}

[1] Belkin, E. A., Poyarkov, V. N. Profilographs of new generation based on holography. "Scientific Review", No. 12. 2015. - pp. 205-208.

[2] Science, education, society: trends and outlooks. Transactions of the Inter. Scientific. Pract. Conf. Part II. August 31, 2013. Ar-Consult. Moscow, 2013. Belkin E. A., Poyarkov V. N. Holographic Profilograph of Passive Control. - pp. 33-35.

[3] Pat. RF No. 2215317. Profilograph /Stepanov Yu. S., Belkin E. A., Barsukov G. V. Appied: 08.01.2002. Published: 27.10.2003. Bull. 30 .

[4] The 2nd Inter. Scientific Conf. Applied Sciences and Europe: Common Challenges and Scientific Findings. September 9-10, 2013. New York, USA. Microrelief Geometrics Simulation and Inspection Tools. Belkin E. A., Poyarkov V. N. pp. 115118.

[5] Energy-saving twenty-first century. Collection of materials $X$ International scientific and practical Internet conference. 01 March - 30 June. Eagle 2012. Geometric modeling of surface microrelief and development of new generation nondestructive testing devices. Y. S. Stepanov, E. A. Belkin, V. Poyarkov N. With 173-178.

[6] Materialy IX miedzynarodowej naukowi-praktycznej konferencji. Wschodnie partnerstwo - 2013. 07 - 15 września 2013 roku. Volume 31. Fizyka. Przemysl. Nauka I studia. 2013. Profiling the new generation. Belkin, E. A., Poyarkov, V. N. 8-10.

[7] Science, education, society: modern challenges and prospects. Collection of scientific papers on the materials of the International scientific-practical conference. Part II. June 28, 2013 AP-consult. Moscow 2013. Inspection tools for control of a microrelief formation process. Belkin E. A., Poyrkov V. N. 117-119.
[8] Science, education, society: trends and prospects. Collection of scientific papers on the materials of the International scientific-practical conference. Part II. August 31, 2013 ARconsult. Moscow 2013. Holographic profilograph of passive control. Belkin, E. A., Poyarkov, V. N.

[9] 2 nd International scientific conference Applied Sciences and Europe: common challenges and scientific findings. 9-10 th August 2013. New York, USA. Microrelief geometrics simulation and inspection tools. Belkin E. A., Poyrkov V. N. P. $115-118$.

[10] Fundamental and applied problems of engineering and technology. FGBOU VPO "state University-unpk" No. 4 (300) 2013 July - Aug. Devices of non-destructive control over the process of formation of topography of the microrelief. Y. S. Stepanov, E. A. Belkin, V. Poyarkov N. C145-148.

[11] Industrial ACS and controllers. No. 4. 2016. Metrological assurance of non-destructive testing of new generation over the formation of the topography of the surface. Belkin, E. A., Poyarkov, V. N. 3-9.

[12] The devices and systems. Management, control, diagnostics. Installation for non-destructive holographic 3D-control. No. 3. 2016. Belkin, E. A., Poyarkov, V. N. From 23-28.

[13] The scientific review. No. 12. 2015. Profiling the new generation on the basis of holography. Belkin, E. A., Poyarkov, V. N. With 205-207.

[14] Belkin, E. A., Poyarkov, V. N., Stepanov. Installation of holographic control over the process of microrelief formation. Modern high-performance technologies and equipment in mechanical engineering. (MTET-2016). Proceedings of the international scientific and technical conference. 6-8.10.2016 St. Petersburg. 